Saúde e Pesquisa, Maringá (PR)

DOI: $10.17765 / 2176-9206.2019 v 12 n 3 p 535-543$

\title{
COMPARAÇÃO ENTRE TRÊS SOFT WARES DE CÁLCULO DE DIETAS: UMA ANÁLISE DO VALOR ENERGÉTICO DE UMA DIETA ENTERAL SEMIARTESANAL
}

Maiane Paris Pirôpo de Oliveira

Discente de nutrição na Universidade Federal do Recôncavo da Bahia, Brasil.

\section{Carlos Alberto Soares da Costa}

Doutor em Ciências. Docente no curso de Nutrição pela Universidade Federal do Recôncavo da Bahia, Brasil.
RESUMO: O objetivo do presente estudo foi recalcular as dietas enterais semiartesanais padrões apresentados em uma Cartilha de Terapia Nutricional Enteral Domiciliar. Foram selecionadas as dietas $1.500,1.800$ e $2.100 \mathrm{kcal}$. Utilizaram-se três softwares de cálculo de dietas, Programa A, B, C, e cálculo manual com o auxílio de três referências bibliográficas. A dieta de $1.500 \mathrm{kcal}$ pelo Programa A apresentou percentual de inadequação de $-22,62 \%$, Programa B $-13,9 \%$, Programa C $+15,73 \%$ e pelo cálculo manual $-1,1 \%$. A dieta de $1.800 \mathrm{kcal}$ apresentou no Programa A -26,6\%, Programa B -15,6\%, Programa $C+11,17 \%$ e no cálculo manual $-1,8 \%$. A dieta de $2.100 \mathrm{kcal}$ pelo Programa A -25,5\%, Programa B -15,8\%, Programa C +7,2\% e no cálculo manual $-4,8 \%$. No estudo foi inviável reproduzir as dietas com auxílio do Programa A, B e C. O cálculo manual foi o método que mais se aproximou dos valores de oferta calórica apresentados pela cartilha.

PALAVRAS-CHAVE: Dieta; Ingestão de energia; Nutrição enteral; Software.

\section{COMPARING THREE SOFT WARES FOR DIET CALCULATION: ANALYSIS OF ENERGETIC VALUE IN A SEMI-AMATEUR ENTERAL DIET}

ABSTRACT: Current analysis recalculates standard semi-amateur enteral diets given in a handout for Home Enteral Nutrition Therapy. Diets with 1,500, 1,800 and 2,100 kcal were selected. Three softwares for diet calculation were employed, namely, Program A, B, C, and a manual calculation, with the help of three bibliographical references. Diet 1,500 kcal of Program A had a non-adequacy percentage of -22.62\%; Program B featured $-13.9 \%$; Program C featured $+15.73 \%$; manual calculation featured $-1.1 \%$. Diet $1,800 \mathrm{kcal}$ features $-6.6 \%$ in Program A; $-15.6 \%$ in Program B; $+11.17 \%$ in Program C; and $-1.8 \%$ in manual calculation. Diet 2,100 kcal of Program A featured -25.5\%; Program B featured $-15.8 \%$; Program $C$ featured $+7.2 \%$; manual calculation featured $-4.8 \%$. Analysis showed unfeasibility of diets with Programs A, $\mathrm{B}$ and $\mathrm{C}$. Manual calculation was the method that was closest to values of calorie calculation presented in the guideline.

KEY WORDS: Diet; Energy intake; Enteral Nutrition; Software.

\section{INTRODUÇÃO}

Autor correspondente:

Carlos Alberto Soares da Costa

nutcarlos@hotmail.com
A terapia nutricional enteral (TNE) trata-se de um conjunto de procedimentos terapêuticos que têm por finalidade fornecer a quantidade necessária de nutrientes para o funcionamento do organismo, visando manter ou recuperar o estado nutricional, por meio 
de via oral, sondas ou ostomias. A TNE é indicada para pacientes, cujo funcionamento do trato gastrointestinal é total ou parcial, porém não conseguem se alimentar por via oral ou quando as necessidades nutricionais não são atingidas somente por esta via ${ }^{2}$. Pode ser administrada em regime hospitalar, ambulatorial ou domiciliar ${ }^{3}$.

Quando indicada e aplicada corretamente, a TNE promove melhoras no estado nutricional do paciente, reduzindo riscos de complicações, taxas de morbimortalidade, tempo de internação, além dos custos hospitalares ${ }^{4}$. Contudo, apesar das inúmeras vantagens da TNE, podem ocorrer diversas complicações decorrentes de sua utilização, dentre elas estão as gastrointestinais, mecânicas e metabólicas5.

A respeito da Terapia Nutricional Domiciliar (TND), na maioria dos casos, a indicação ocorre durante o período de internação hospitalar, possibilitando ao paciente a continuidade do tratamento em seu domicílio $^{6}$. A TND compreende a Terapia Nutricional Enteral Domiciliar (TNED), Terapia Nutricional Parenteral Domiciliar (TNPD) e Suplemento Oral Domiciliar (SOD)7. Sua prática é relevante, uma vez que oferece inúmeras vantagens, com destaque ao ambiente familiar confortável, propiciando um tratamento humanizado, melhor qualidade de vida ao paciente, redução de complicações clínicas e nutricionais, menor chance de reinternações precoces, além de contribuir para redução dos custos hospitalares ${ }^{7.9}$.

ATNED pode ser conduzida por meio de fórmulas industrializadas ou não industrializadas, usualmente denominadas de artesanais, compostas por alimentos in natura ou processados ${ }^{3}$. De acordo com a Sociedade Brasileira de Nutrição Parenteral e Enteral (SBNPE) 7, as dietas industrializadas garantem o fornecimento adequado dos nutrientes na sua totalidade, é segura, possui maior controle microbiológico, evita obstrução do dispositivo e tem viscosidade adequada. A TN mista, na qual se intercala uso de dietas industrializadas com preparações "caseiras", pode ser utilizada desde que se avaliem alguns critérios específicos, tais como a presença de gastrostomia, moradia com boas condições higiênicosanitária, não apresentar quadro de desnutrição, não possuir necessidade energética acima de $2.000 \mathrm{kcal}$, não possuir lesão por pressão, monitoramento por parte da equipe multiprofissional ${ }^{6}$. Apesar das dietas industrializadas serem mais aceitas na prática clínica, muitas vezes sua utilização torna-se inviável para pacientes que necessitam dar continuidade ao tratamento em longo prazo, pelo elevado custo financeiro ${ }^{8}$. Nestes casos uma alternativa são as dietas artesanais ou semiartesanais, pois além de apresentarem menor custo e fácil preparo, possibilitam realizar modificações em sua formulação, conforme a tolerância e preferência do paciente. Desta forma, promove um suporte nutricional individualizado, permitindo a seleção de alimentos convencionalmente consumidos pelo paciente9. Contudo, apesar dessas vantagens, possuem pouco controle microbiológico, menor instabilidade físico-química e imprecisão das propriedades nutricionais ${ }^{10-11}$.

Diante da importância da TNED para manter ou recuperar o estado nutricional dos pacientes, foi divulgada, em 2018, a segunda edição da Cartilha de Terapia Nutricional Enteral/ Manual do Paciente Cuidador ${ }^{12}$. A publicação foi elaborada pela Secretaria Municipal de Saúde de Belo Horizonte, por meio da Secretaria Municipal de Saúde, com o apoio da Universidade Federal de Minas Gerais (UFMG) ${ }^{12}$.

Areferida cartilha trazinformações sobre cuidados no preparo da dieta enteral, conservação, modos de administração, limpeza da sonda, hidratação do paciente, procedimentos em situações de entupimento da sonda, diarreia, constipação intestinal, náuseas e vômitos, estase gástrica e saída da sonda. Ainda traz informações sobre outros cuidados com o paciente ${ }^{13}$. Ao final da publicação, é apresentada uma proposta de dieta artesanal com formulações de $1.500,1.800$ e 2.100 quilocalorias e as opções: padrão ou para diabéticos ${ }^{13}$. As formulações foram elaboradas pensando-se em uma dieta nutricionalmente completa e de baixo custo e as composições nutricionais das dietas foram avaliadas quimicamente, por meio de análises bromatológicas dos macros e micronutrientes ${ }^{14}$.

Considerando a importância da oferta adequada de nutrientes para recuperação do estado nutricional dos pacientes, faz-se necessário reproduzir as dietas propostas pela cartilha da UFMG, uma vez que a forma de preparo, sazonalidade, regionalização, dentre outros fatores, podem influenciar diretamente na composição nutricional da dieta. Além disso, a padronização dos 
cálculos dietéticos através do sistema informatizado possibilita reajustar o plano alimentar de acordo com as complicações que o paciente venha ter ou desenvolver durante a TNED.

Diante do exposto, o presente trabalho teve por objetivo recalcular as dietas enterais artesanais padrões, apresentadas na Cartilha de Terapia Nutricional Enteral/ Manual do Paciente Cuidador, proposta pela UFMG, utilizando como método softwares de cálculos de dietas e cálculo manual, no intuito de verificar a adequação de acordo com os valores apresentados pela cartilha.

\section{METODOLOGIA}

O presente estudo foi realizado no período de 10 de agosto de 2018 a 27 de maio de 2019. Os cálculos foram realizados no laboratório de informática da Instituição de Ensino. Foram selecionadas as dietas enterais semiartesanal padrão de $1.500,1.800$ e 2.100 quilocalorias, apresentadas na Cartilha de Terapia Nutricional Enteral/ Manual do Paciente Cuidador UFMG.

Utilizaram-se três softwares de cálculo de dietas, o Programa A e B possuem versão gratuita disponível na internet para teste, o Programa $C$ versão 4.0 possui licença adquirida pela instituição de ensino. Além dos programas, realizou-se cálculo manual utilizando como referências a tabela brasileira de composição dos alimentos ${ }^{15}$, a tabela para avaliação de consumo alimentar em medidas caseiras ${ }^{16}$ e a tabela de composição dos alimentos $^{17 .}$

Em cada programa computacional, várias tabelas de composição de alimentos (TCAs) abastecem seus bancos de dados. Durante as análises preconizou-se uma ordem de prioridade para o uso das TCAs, a fim de minimizarem os erros após a comparação dos resultados. A ordem sequencial adotada foi: tabela brasileira de composição dos alimentos ${ }^{15}$, tabela de composição dos alimentos ${ }^{17}$ e as tabelas de composição nutricional dos alimentos consumidos no Brasil, do Instituto Brasileiro de Geografia e Estatistica ${ }^{18 .}$ No Programa A não consta a Tabela para avaliação de consumo alimentar em medidas caseiras $^{16}$ e no Programa B apenas para cadastro das medidas.
Para análise dos resultados, utilizou-se como parâmetros a oferta de energia apresentada nas dietas semiartesanais da UFMG. Os resultados foram expressos em percentuais em relação ao valor de referência, para verificar o quanto se distanciam do valor da cartilha.

\section{RESULTADOS}

A dieta de $1.500 \mathrm{kcal}$ (Tabela 1), pelo Programa A, apresentou oferta energética de 1.160,8 kcal (-22,62\%), pelo Programa B, 1.290,1 kcal (-13,9\%), Programa C $1.735,95 \mathrm{kcal}(+15,73 \%)$ e pelo cálculo manual $1.483,41$ kcal (-1,1\%).

A dieta de $1.800 \mathrm{kcal}$ (Tabela 2) apresentou no Programa A 1.322,8 kcal (-26,6\%), no Programa B 1.517,5 kcal (-15,6\%), Programa C 2.001,9 kcal, (+11,17\%) e no cálculo manual 1.766,02 kcal (-1,8\%).

A dieta de $2100 \mathrm{kcal}$ (Tabela 3) pelo Programa A apresentou 1.563,88 kcal (-25,5\%), pelo Programa B $1.563,88 \mathrm{kcal}(-15,8 \%)$, Programa C $2.252,34 \mathrm{kcal}(+7,2$ \%), e no cálculo manual 1.997,82 kcal (-4,8\%). 
Tabela 1. Cálculo e análise da oferta calórica de uma dieta enteral semiartesanal de $1.500 \mathrm{Kcal}$ com o auxílio de três programas computacionais, Santo Antônio de Jesus-Ba, 2019

\begin{tabular}{lccccc}
\hline \multirow{2}{*}{ Ingredientes } & 1500 kcaf & Programa A* $^{*}$ & Programa B** & Programa C $^{\S}$ & Cálculo manual $^{\dagger}$ \\
\cline { 2 - 5 } & Qtde de ingredientes & Calorias (kcal) & Calorias (kcal) & Calorias (kcal) & Calorias (kcal) \\
\hline Leite desnatado (ml) & 1000 & 0,00 & 0,00 & 419,90 & 350,00 \\
Ovo (g) & 45 (2x/semana) & 65,57 & 156,00 & 65,18 & 65,70 \\
Albumina em pó pura (g) & 17,4 (5x/semana) & 60,03 & 61,00 & 62,64 & 11,96 \\
Farinha de aveia (g) & 45 & 172,80 & 170,00 & 171,90 & 171,00 \\
Creme de arroz enriquecido (g) & 50 & 193,00 & 186,30 & 187,20 & 193,00 \\
Batata inglesa (g) & 280 & 145,45 & 146,00 & 248,68 & 145,60 \\
Castanha-do-Pará (g) & 02 & 13,12 & 14,00 & 13,68 & 13,12 \\
Óleo de canola (mL) & 26 & 208,00 & 234,00 & 234,00 & 229,84 \\
Óleo de soja (mL) & 13 & 114,90 & 117,00 & 117,00 & 114,92 \\
Cereal comercial à base de milho (g) & 05 & 18,27 & 18,30 & 18,65 & 18,25 \\
Canela em pó (g) & 12 & 31,32 & 43,00 & 43,62 & 31,32 \\
Açúcar cristal (g) & 13,8 & 53,38 & 54,5 & 55,18 & 53,40 \\
Sal iodado (g) & 02 & 0,00 & 0,00 & 0,00 & 0,00 \\
Cenoura, crua (g) & 55 & 18,77 & 19,00 & 25,42 & 18,70 \\
Laranja Pera Rio, suco (g) & 180 & 66,19 & 71,00 & 72,90 & 66,60 \\
\hline Total (kcal) & & $1.160,80$ & $1.290,10$ & $1.735,95$ & $1.483,40$ \\
\hline
\end{tabular}

${ }^{\ddagger}$ Cartilha de Terapia Nutricional Enteral/ Manual do Paciente Cuidador, proposta pela Universidade Federal de Minas Gerais ${ }^{13 ; *}$ https:// dietbox.me/pt-BR/home; **http://www.dietsmartsystem.com/DietSmart/; ${ }^{\S} \mathrm{https}: / /$ www.avanutri.com.br/software_avanutri_online; ${ }^{\dagger}$ Referências usadas para o cálculo manual: Tabela brasileira de composição de alimentos ${ }^{15}$, PINHEIRO et al. ${ }^{16}$, PHILIPPII ${ }^{17}$.

Tabela 2. Cálculo e análise da oferta calórica de uma dieta enteral semiartesanal de 1.800 kcal com o auxílio de três programas computacionais, Santo Antônio de Jesus-BA, 2019

(Continua)

\begin{tabular}{|c|c|c|c|c|c|}
\hline \multirow{2}{*}{ Ingredientes } & $1800 \mathrm{kcal}^{+}$ & Programa A* & Programa $B^{* * *}$ & Programa $C^{\S}$ & Cálculo manual $^{\dagger}$ \\
\hline & Qtde de ingredientes & Calorias (kcal) & Calorias (kcal) & Calorias (kcal) & Calorias (kcal) \\
\hline Leite desnatado (mL) & 500,00 & 0,00 & 0,00 & 209,95 & 175,00 \\
\hline Leite de vaca integral $(\mathrm{mL})$ & 500 & 0,0 & 63,00 & 308,80 & 295,00 \\
\hline Ovo (g) & 45 (2x/semana) & 65,57 & 156,00 & 65,18 & 65,70 \\
\hline Albumina em pó pura (g) & $17,4(5 x /$ semana $)$ & 60,03 & 61,00 & 62,64 & 11,96 \\
\hline Farinha de aveia (g) & 45 & 172,80 & 170,00 & 171,90 & 171,00 \\
\hline Creme de arroz enriquecido (g) & 55 & 212,30 & 205,00 & 205,92 & 212,30 \\
\hline Batata inglesa (g) & 280 & 145,45 & 146,00 & 248,68 & 145,60 \\
\hline Castanha-do-Pará (g) & 02 & 13,12 & 14,00 & 13,68 & 13,12 \\
\hline Óleo de canola (mL) & 26 & 208,00 & 234,00 & 234,00 & 229,84 \\
\hline Óleo de soja (mL) & 13 & 114,90 & 117,00 & 117,00 & 114,92 \\
\hline Cereal comercial à base de milho (g) & 15 & 54,80 & 54,90 & 55,90 & 54,75 \\
\hline Canela em pó (g) & 12 & 31,12 & 43,00 & 43,62 & 31,32 \\
\hline Açúcar cristal (g) & 27,6 & 106,77 & 109,00 & 110,32 & 106,81 \\
\hline
\end{tabular}


(Conclusão)

\begin{tabular}{|c|c|c|c|c|c|}
\hline \multirow{2}{*}{ Ingredientes } & $1800 \mathrm{kcal}^{\ddagger}$ & Programa A* & Programa $B^{* * *}$ & Programa $C^{\S}$ & Cálculo manual $^{\dagger}$ \\
\hline & Qtde de ingredientes & Calorias (kcal) & Calorias (kcal) & Calorias (kcal) & Calorias (kcal) \\
\hline Sal iodado (g) & 02 & 0,00 & 0,00 & 0,00 & 0,00 \\
\hline Cenoura, crua (g) & 55 & 18,17 & 19,00 & 25,42 & 18,70 \\
\hline Laranja Pera Rio, suco (g) & 180 & 66,19 & 71,00 & 72,90 & 66,60 \\
\hline Açúcar cristal (g) & 13,80 & 53,38 & 54,50 & 55,18 & 53,40 \\
\hline \multirow[t]{2}{*}{ Total (Kcal) } & & $1.322,60$ & $1.517,50$ & $2.001,09$ & $1.766,02$ \\
\hline & & $(-26,6 \%)$ & $(-15,6 \%)$ & $(+11,17 \%)$ & $(-1,8 \%)$ \\
\hline
\end{tabular}

Cartilha de Terapia Nutricional Enteral/ Manual do Paciente Cuidador, proposta pela Universidade Federal de Minas Gerais $13 ;$ * https://dietbox me/pt-BR/home; **http://www.dietsmartsystem.com/DietSmart/; $\$$ https://www.avanutri.com.br/software avanutri_online; ${ }^{\dagger}$ Referências usadas para o cálculo manual: Tabela brasileira de composição de alimentos ${ }^{15}$, PINHEIRO et al. ${ }^{16}$, PHILIPPI ${ }^{17}$.

Tabela 3. Cálculo e análise da oferta calórica de uma dieta enteral semiartesanal de 2.100 kcal com o auxílio de três programas computacionais, Santo Antônio de Jesus-Ba, 2019

(Continua)

\begin{tabular}{|c|c|c|c|c|c|}
\hline \multirow{2}{*}{ Ingredientes } & $2100 \mathrm{kcal}^{\ddagger}$ & Programa A* & Programa $B^{* * *}$ & Programa $C^{\S}$ & Cálculo manual $^{\dagger}$ \\
\hline & Qtde de ingredientes & Calorias (kcal) & Calorias (kcal) & Calorias (kcal) & Calorias (kcal) \\
\hline Leite desnatado (mL) & 500 & 0,00 & 0,00 & 209,95 & 175,00 \\
\hline Leite de vaca integral $(\mathrm{mL})$ & 500 & 0,00 & 63,00 & 308,80 & 295,00 \\
\hline Ovo (g) & 45 (2x/semana) & 65,57 & 156,00 & 65,18 & 65,70 \\
\hline Albumina em pó pura (g) & 29 (5x/semana) & 104,05 & 103,00 & 104,40 & 19,93 \\
\hline Farinha de aveia (g) & 45 & 172,80 & 170,00 & 171,90 & 171,00 \\
\hline Creme de arroz enriquecido (g) & 55 & 212,30 & 205,00 & 205,92 & 212,30 \\
\hline Batata inglesa (g) & 280 & 145,45 & 146,00 & 248,68 & 145,60 \\
\hline Castanha-do-Pará (g) & 02 & 13,12 & 14,00 & 13,68 & 13,12 \\
\hline Óleo de canola (mL) & 26 & 208,00 & 234,00 & 234,00 & 229,84 \\
\hline Óleo de soja (mL) & 26 & 221,00 & 234,00 & 234,00 & 229,84 \\
\hline Cereal comercial à base de milho (g) & 25 & 91,34 & 91,50 & 93,25 & 91,25 \\
\hline Canela em pó (g) & 12 & 31,32 & 43,00 & 43,62 & 31,32 \\
\hline Açúcar cristal (g) & 27,6 & 106,77 & 109 & 110,32 & 106,81 \\
\hline Sal iodado (g) & 02 & 0,00 & 0,00 & 0,00 & 0,00 \\
\hline Cenoura, crua (g) & 55 & 18.77 & 19,00 & 25,42 & 18,70 \\
\hline Laranja Pera Rio, suco (g) & 180 & 66,19 & 71,00 & 72,90 & 66,60 \\
\hline Açúcar cristal (g) & 27,6 & 106,77 & 109,00 & 110,32 & 106,81 \\
\hline \multirow[t]{2}{*}{ Total (Kcal) } & & $1.563,88$ & $1.767,50$ & $2.252,34$ & 1997,82 \\
\hline & & $(-25,52 \%)$ & $(-15,8 \%)$ & $(+7,2 \%)$ & $(-4,8 \%)$ \\
\hline
\end{tabular}

Cartilha de Terapia Nutricional Enteral/Manual do Paciente Cuidador, proposta pela Universidade Federal de Minas Gerais $13 ; *$ https://dietbox me/pt-BR/home; **http://www.dietsmartsystem.com/DietSmart/; $\&$ https://www.avanutri.com.br/software avanutri_online; ${ }^{\dagger}$ Referências usadas para o cálculo manual: Tabela brasileira de composição de alimentos ${ }^{15}$, PINHEIRO et al. ${ }^{16}$, PHILIPPI $^{17}$. 


\section{DISCUSSÃO}

Os resultados demonstraram divergências na quantidade de energia calculada pelas quatro ferramentas, no entanto é possível destacar que o cálculo manual foi a ferramenta que mais se aproximou dos valores apresentados na cartilha da UFMG. Os cálculos realizados com auxílio do Programa A e B favorecem o maior risco de perda ponderal, visto que proporcionaram menor oferta de energia. Enquanto o Programa C favorece maior risco para ganho ponderal, pela maior oferta de energia.

A alimentação é um fator essencial na conservação da saúde, bem como na prevenção e tratamento de doenças $^{19}$. É importante destacar que os pacientes em uso de TNED podem encontrar-se já desnutridos, pelas complicações associadas à doença de base/ou tratamento, ou tornarem-se desnutridos durante a atenção domiciliar ${ }^{20}$. Diversos são os fatores que contribuem para desnutrição, contudo o consumo alimentar inadequado tem sido apontado como fator importante para evolução deste quadro clínico ${ }^{21}$. Dentre as consequências da desnutrição estão as novas internações hospitalares, bem como o alto índice de morbimortalidades ${ }^{22}$.

Por outro lado, o consumo alimentar excessivo, juntamente com o sedentarismo, pode favorecer ao sobrepeso e a obesidade, os quais são considerados mundialmente um problema de saúde pública ${ }^{23}$. Pacientes em uso de TNED, normalmente pela condição clínica encontram-se acamados apresentando menor gasto energético, fato este que associado à alta oferta de energia pode favorecer ao ganho de peso inadequado. A obesidade é um importante fator de risco para o desenvolvimento de outras doenças crônicas não transmissíveis (DCNT), como doenças cardiovasculares, diabetes melitus tipo 2 , cânceres ou mortalidade geral ${ }^{2425}$.

É importante destacar que não foi objetivo do presente trabalho verificar as inadequações existentes nos softwares de cálculos de dietas, senão, padronizar os cálculos para fazer possíveis ajustes caso os pacientes necessitem de TNED mais individualizada. Contudo, com o desfecho do trabalho foi possível observar que, apesar do sistema informatizado permitir de forma prática e rápida o cálculo do consumo alimentar, as fontes de referências que alimentam suas bases de dados podem gerar resultados divergentes e não fidedignos, comprometendo a prescrição ou a análise dietética ${ }^{26}$.

As divergências encontradas no presente estudo, alerta para maior atenção no uso das TCAs, que compõem o banco de dados dos softwares de cálculo de dietas, pois de acordo com Drehmer et al. ${ }^{27}$, há mais de uma década existe uma lacuna com relação a pesquisas e publicações sobre os nutrientes presentes nos alimentos brasileiros; as tabelas existentes não apresentam, em sua maioria, dados completos, não descrevem os métodos utilizados para análise, não informam o estado físico do alimento, se cru ou preparado, sua procedência, nem a composição e origem das receitas. Desta forma, diferentes tabelas podem apresentar resultados discrepantes sobre o mesmo alimento, influenciando diretamente na análise dietética.

Os resultados obtidos no presente estudo corroboram com os achados encontrados por outros autore ${ }^{28,29,30}$, sinalizando para atenção no uso destas ferramentas. Em estudo, que comparou dois programas computacionais utilizados na estimativa do consumo alimentar de crianças, concluiu-se que os programas selecionados para análise do consumo dietético, apresentaram diferenças quanto às gramaturas utilizadas, com reflexos importantes na estimativa de energia e nutrientes do consumo alimentar infantil ${ }^{28}$.

Outro estudo ${ }^{29}$, ao comparar três programas computacionais utilizados na avaliação de recordatórios alimentares 24 horas, verificou também divergências entre os programas computacionais analisados, sugerindo melhorias referentes à análise dietética, padronização das medidas caseiras e a confiabilidade dos dados de composição alimentar inseridas nos programas.

No estudo realizado por Ramoni et al..$^{30}$, foi calculado o consumo alimentar a partir de duas TCAs e foram encontradas divergências significativas entre os valores nutricionais referente à quantidade de energia e nutrientes. O cálculo manual, utilizando as TCAs apesar de ser mais trabalhoso e demandar maior tempo, no presente trabalho foi o que apresentou melhor resultado comparado aos Programas A, B e C. Assim os resultados do presente estudo alertam para o uso dos instrumentos disponíveis para cálculo de dietas e a sua influência na prescrição nutricional. 
Dentre as limitações do presente estudo destacase a dificuldade de encontrar TCAs completas, que possuam todos os alimentos. Para realizar os cálculos, tanto manual como pelos softwares, foi necessário selecionar várias tabelas e manter uma ordem sequencial de utilização, pois o mesmo alimento calculado por diferentes tabelas apresentou resultados divergentes. Em nenhuma das tabelas ${ }^{15,16,17,18}$ foi encontrado o ingrediente albumina, porém os programas trazem as informações nutricionais do fabricante.

Os dados obtidos no presente estudo evidenciam a importância de conhecer os métodos utilizados pelas TCAs para determinar a composição nutricional dos alimentos. Uma vez que diversos fatores como utilização de métodos analíticos impróprios, amostragem inadequada, descrição incorreta de alimentos e/ou fontes de valores nutricionais, procedência, estado físico e forma de preparo, podem influenciar diretamente em sua composição nutricional ${ }^{27}$.

No estudo de Ribeiro et al.,31 avaliou-se a concordância entre os valores de macronutrientes e energia de alimentos analisados em laboratório com os dados apresentados em tabelas e softwares de composição de alimentos em uso no Brasil. Os resultados demostraram diferenças significativas quando comparados entre si. Os autores ressaltam que a análise laboratorial seria a forma mais adequada de realizar a avaliação da composição nutricional dos alimentos, porém o método possui alto custo, demanda estrutura adequada e maior tempo para análise. Desta forma, o uso das TCAs e softwares tornam-se mais viável, por estar facilmente disponível, para exercer tais funções.

Outra limitação refere-se à escassez de estudos que abordam o tema, pois a maioria das pesquisas disponíveis na literatura ${ }^{28,29,31}$ possui mais de cinco anos de publicação. Estudos dessa natureza poderão contribuir para aprimorar o delineamento dos programas computadorizados e melhorar a confiabilidade no uso dessa ferramenta que é tão útil na prática clínica. Entretanto, deve-se ter cautela na continuidade do uso destes softwares. Como observado no presente estudo, há diferenças nutricionalmente significativas entre os resultados calculados pelos TCAs e softwares comparados aos analisados em laboratório.
Conhecer a composição nutricional dos alimentos é de extrema importância na prática clínica, pois, auxilia na tomada de decisões para manter ou recuperar o estado nutricional do paciente em uso de TNED ${ }^{32}$. Contudo, fazse necessário que as tabelas de composição química dos alimentos que compõem a base de dados dos softwares sejam atualizadas, complementadas e revisadas a fim de fornecerem informações mais precisas e confiáveis.

\section{CONCLUSÃO}

Com base nos resultados do presente estudo, foi inviável reproduzir as dietas da cartilha da UFMG com auxílio dos Programas A, B e C, uma vez que os mesmos apresentam valores divergentes, podendo comprometer o estado nutricional dos pacientes em uso de TNED. Além disso, o cálculo manual foi o método que mais se aproximou dos valores de oferta calórica apresentados pela cartilha. Diante dos resultados, sugerem-se atualizações das TCAs, bem como nos softwares de cálculos de dietas, no intuito de obter maior adequação dos resultados e confiabilidade na análise do consumo alimentar.

\section{REFERÊNCIAS}

1. Singer P. Preserving the quality of life: nutrition in the ICU. Crit. Care. 2019; 23(Suppl 1): 139.

2. Limketkai BN, Shah ND, Sheikh GN, Allen K. Classifying Enteral Nutrition: Tailored for Clinical Practice. Curr. Gastroenterol Rep. 2019; 21(9): 47.

3. Pash E. Enteral Nutrition: Options for Short-Term Access. Nutr. Clin. Pract. 2018; 33(2): 170-176.

4. Kurien M, Williams J, Sanders DS. Malnutrition in healthcare settings and the role of gastrostomy feeding. Proc. Nutr. Soc. 2017; 76(3): 352-360.

5. Wanden-Berghe C, Patino-Alonso MC, Galindo-Villardón P, Sanz-Valero

J.Nutrients. Complications Associated with Enteral Nutrition: CAFANE Study. Nutrients. 2019; 11(9): E2041.

6. Mazur CE, Zago RCC, Schieferdecker MEM, Maluf EMCP. Home enteral nutrition: clinical-nutritional 
analysis and outcomes of 10 years of public policy. Nutr. Hosp. 2019; 36(4): 758-763.

7. Sociedade Brasileira de Nutrição Parenteral e Enteral. Diretriz Brasileira de Terapia Nutricional Domiciliar. BRASPEN J 2018; 33 (Supl 1): 37-46.

8. Hyeda A, Costa ESMD.

Economic analysis of costs with enteral and parenteral nutritional therapy according to disease and outcome. Einstein (Sao Paulo). 2017; 15(2): 192.

9. Maniglia FP, Pagnani, ACC, Nascimento GG. Desenvolvimento de dieta enteral artesanal com propriedades funcionais. Rev. Bras. Nutr. Clin. 2015; 30 (1): 66-70.

10. Vasconcelos C, Fornari JV, Arçari DP, Bernabe AS, Leonardo MJ, Ferraz RR. Comparação entre dieta industrializada e dieta caseira em relação aos custos e contaminações microbiológicas. Rev. Saúde em Foco 2013; 7: 41-44.

11. Bobo E. Reemergence of blenderized tube feedings. Nutrition in Clinical Practice 2016; 31(6): 730-735.

12. Jansen AK, Henriques GS, Miranda LAG, Guedes EG, Rodrigues AMS, Generoso SV. Terapia nutricional enteral domiciliar: promoção do direito humano à alimentação adequada. $7^{\circ}$ Congresso Brasileiro de Extensão Universitária. 07 a 09 de setembro de 2011. ISBN: 978-85-93416-00-2.

13. Cartilha de Terapia Nutricional enteral Manual do Paciente / Cuidador. Belo Horizonte 2018. Disponível em: https://prefeitura.pbh.gov.br/sites/ default/files/estrutura-de-governo/saude/2018/ documentos/publicacoes\%20atencao\%20saude/ Cartilha_terapia_nutricional_enteral-25-6-2018.pdf Acesso: $22 / 03 / 2019$.

14. Jansen AK, Henriques GS, Miranda, LA, Guedes EG, Rodrigues AMS, Generoso SV. Relato de experiência: terapia nutricional enteral domiciliar - promoção do direito humano à alimentação adequada para portadores de necessidades alimentares especiais. Rev. Demetra; 2014; 9(Supl.1): 233-247.

15. Tabela brasileira de composição de alimentos. NEPA, UNICAMP. 4. ed. rev. e ampl. Campinas: NEPAUNICAMP, 2011. $161 \mathrm{p}$.
16. Pinheiro ABV, Lacerda EMA, Benzecry EH, Gomes MCS, Costa VM. Tabela para avaliação de consumo alimentar em medidas caseiras. $5^{\mathrm{a}}$. ed. São Paulo: Atheneu, 2004. $131 \mathrm{p}$.

17. Philippi ST. Tabela de composição de alimentos: suporte para decisão nutricional. $6^{\mathrm{a}}$. ed. São Paulo: Coronário; 2017.

18. Instituto Brasileiro de Geografia. Tabelas de Composição Nutricional dos Alimentos Consumidos no Brasil. IBGE -2011. Disponível em: https:// ww2.ibge.gov.br/home/estatistica/populacao/ condicaodevida/pof/2008 2009 composicao nutricional/default_zip.shtm. Acesso: 27/04/19.

19. Di Renzo L, Gualtieri P, Romano L, Marrone G, Noce A, Pujia A, Perrone MA, Aiello V, Colica C, De Lorenzo A. Role of Personalized Nutrition in Chronic-Degenerative Diseases. Nutrients. 2019; 24; 11(8): E1707.

20. Leydon N, Dahl W. Improving the nutritional status of elderly residents of long-term care homes. J. Health Serv. Res. Policy. 2008; 13: 25-29.

21. Curtis LJ, Bernier P, Jeejeebhoy K, Allard J, Duerksen D, Gramlich L, Laporte M, Keller HH. Costs of hospital malnutrition. Clin. Nutr. 2017; 36(5): 1391-1396.

22. Simmons SF, Keeler E, Zhuo X, Hickey KA, Sato HW, Schnelle JF. Prevention of unintentional weight loss in nursing home residents: a controlled trial of feeding assistance. J. Am. Geriatr. Soc. 2008; 56 : 1466-1473.

23. Engin A. The Definition and Prevalence of Obesity and Metabolic Syndrome. Adv. Exp. Med. Biol. 2017; 960: 1-17.

24. Cuppari L. Nutrição clínica no adulto. 4ed. São Paulo: Manole, 2019.

25. Kuroda M, Sakaue H. Adipocyte Death and Chronic Inflammation in Obesity. J. Med. Invest. 2017; 64(3.4): 193-196.

26. Vieira FGK, Pietro PF, Feio LC, Assis MAA, Peres MA, Vasconcelos FAG. Comparação do valor nutricional de dez cardápios segundo quatro programas computacionais. Rev. Nutr. 2009; 22(1): 29-38.

27. Drehmer M, Melere C, Reinheimer SM, Camey SA. 
Agreement of dietary fiber and calorie intake values according to the choice of nutrient composition and household measure tables. Rev. Nutr. 2017; 30: 233- 244 .

28. Costa RS, Antunes MML, Mello MA, Sichieri R. Comparação de dois programas computacionais utilizados na estimativa do consumo alimentar de criança. Rev. Bras. Epidemiol. 2007; 10(2): 267-275.

29. Lourenço PKAC, Castro JL, Vale SHL, Alves CX, Leite LD. Comparação de três programas computacionais utilizados na avaliação de recordatórios alimentares 24 horas. J. Health. Inform. 2011; 3(1): 13-8.

30. Romani CTC, Lima EC, Closs VE, Macagnan FE, Oliveira MS, Gustavo AS, Feoli AMP. Comparação do consumo alimentar calculado a partir de duas tabelas de composição de alimentos. Saúde e Pesquisa 2019; 12 (1): 19-27.

31. Ribeiro P, Morais TB, Colugnati FAB, Sigulem DM. Tabelas de composição química de alimentos: análise comparativa com resultados laboratoriais. Rev. Saúde Pública vol.37 no.2 São Paulo Apr. 2003.

32. Koekkoek KW, van Zanten AR. Nutrition in the critically ill patient. Curr. Opin. Anaesthesiol. 2017;30(2):178-185.

Recebido em: 10/06/2019

Aceito em: 11/09/2019 